\title{
The Instrumented Fetal Sheep as a Model of Cerebral White Matter Injury in the Premature Infant
}

\author{
Stephen A. Back • Art Riddle • Justin Dean • \\ A. Roger Hohimer
}

Published online: 8 March 2012

(C) The American Society for Experimental NeuroTherapeutics, Inc. 2012

\begin{abstract}
Despite advances in neonatal intensive care, survivors of premature birth remain highly susceptible to unique patterns of developmental brain injury that manifest as cerebral palsy and cognitive-learning disabilities. The developing brain is particularly susceptible to cerebral white matter injury related to hypoxia-ischemia. Cerebral white matter development in fetal sheep shares many anatomical and physiological similarities with humans. Thus, the fetal sheep has provided unique experimental access to the complex pathophysiological processes that contribute to injury to the human brain during successive periods in development. Recent refinements have resulted in models that replicate major features of acute and chronic human cerebral injury and have provided access to complex clinically relevant studies of cerebral blood flow and neuroimaging that are not feasible in smaller laboratory animals. Here, we focus on emerging insights and methodologies from studies in fetal sheep that have begun to define cellular and vascular factors that contribute to white matter injury. Recent advances include spatially defined measurements of cerebral blood flow in utero, the definition of cellular maturational factors that define the topography of injury and the application of high-field magnetic resonance imaging to
\end{abstract}

S. A. Back $\cdot$ A. Riddle $\cdot$ J. Dean

Department of Pediatrics, Oregon Health Sciences University, Portland, OR 97239, USA

\section{S. A. Back $(\square)$}

Departments of Pediatrics and Neurology,

Oregon Health Sciences University,

Portland, OR 97239, USA

e-mail: backs@ohsu.edu

\section{A. R. Hohimer}

Department of Obstetrics and Gynecology, Oregon Health

Sciences University,

Portland, OR 97239, USA define novel neuroimaging signatures for specific types of chronic white matter injury. Despite the higher costs and technical challenges of instrumented preterm fetal sheep models, they provide powerful access to clinically relevant studies that provide a more integrated analysis of the spectrum of insults that appear to contribute to cerebral injury in human preterm infants.

Keywords Myelin - Oligodendrocyte - White matter . Ovine $\cdot$ Hypoxia-ischemia $\cdot$ Cerebral blood flow $\cdot$ MRI . Cerebral palsy

\section{Overview of the Clinical Problems Accessible in Preterm Fetal Sheep}

The last decade has seen a resurgence of interest in instrumented preterm fetal sheep preparations to study the complex pathophysiological processes that contribute to brain injury in the preterm infant. The merits of a large pre-clinical animal model have become increasingly recognized, as it has become apparent that rodent models have significant limitations to study injury to the developing human brain [1]. Not only do preterm fetal sheep preparations closely replicate major features of acute and chronic human preterm brain injury, but they also provide access to complex, clinically relevant studies of cerebral blood flow and neuroimaging that are not feasible in smaller laboratory animals.

Fetal sheep preparations have provided powerful access to large animal pre-clinical testing of neuroprotective agents for the treatment of neonatal encephalopathies related to hypoxia-ischemia or maternal fetal infection. It is increasingly recognized that the selection of appropriate animal models is critical to identify promising therapeutic agents and to reduce false negative outcomes [2]. The substantial 
translational advantages of studies in a large preclinical animal model are exemplified by pre-clinical studies in near term fetal sheep [3] that lead to the head cooling trials for neonatal encephalopathy, and the implementation of cerebral hypothermia protocols in neonatal intensive care units worldwide [4].

In recent years, studies of hypoxic-ischemic injury to the developing brain have yielded an increasingly complex and controversial set of observations related to the pathogenetic mechanisms that result in injury to white and gray matter structures within the neuraxis. We will focus on approaches to study cerebral white matter injury (WMI), the major form of brain injury, and the leading cause of chronic neurological disability in survivors of premature birth [5]. The broader questions of the pathogenetic mechanisms that relate WMI to cortical and subcortical gray matter injury have been recently reviewed [6].

Brain injury in preterm survivors has an unexplained predilection for cerebral white matter. The period of highest risk for WMI is $\sim 23$ to 32 weeks postconceptional age. Although major advances in the care of premature infants have resulted in striking improvements in the survival of very low birth weight infants $(<1.5 \mathrm{~kg})$, improved survival has been accompanied by a significant increase in the number of pre-term survivors with long-term neurological deficits [7]. The major consequences of this injury are permanent motor impairment (i.e., cerebral palsy [CP]) ranging from mild to profound spastic motor deficits [8-13], as well as a broad spectrum of cognitive, social behavioral, attentional, visual, and learning disabilities that manifest by school age in 25 to $50 \%$ of children [14-18]. In preterm survivors, magnetic resonance image (MRI)-defined WMI, but not gray matter injury manifests in the first months of life as abnormal movements that are predictive of CP [19-21]. The impact of WMI can be appreciated from a recent large population-based study of children with CP. Perinatal WMI (including periventricular leukomalacia) was the most common finding, which was seen in almost half (42.5\%) of the affected children [22]. WMI is not exclusively associated with prematurity and it is increasingly appreciated in term infants [23-25]. Infants with complex congenital heart disease are at particular risk for WMI and delayed brain maturation [26-28]. Because very low birth weight infants comprise approximately $1.5 \%$ of the 4 million live births in the United States alone each year, the worldwide social and economic burden is considerable. The average lifetime costs per person with $\mathrm{CP}$ is estimated to be $\sim 1$ million dollars in the United States. [29]. An understanding of the cellular and molecular basis of preterm WMI is thus urgently needed to develop effective interventions to prevent these lifelong neurological disabilities. Hence, there continues to be a critical need for suitable animal models that permit hypothesis-driven studies of the pathogenesis of WMI in human preterm survivors.

\section{Advantages and Disadvantages of the Fetal Sheep to Model WMI}

Fetal sheep preparations require substantial cost and infrastructure to support the surgical instrumentation and postoperative care of large laboratory animals. Fetal sheep studies require a highly skilled surgical team, a large animal operating facility suitable for sterile operations, specialized veterinary care, and access to reliable breeders. Despite these challenges, many preparations have yielded very reproducible results with low morbidity and mortality, thereby limiting the number of animals required. Presently, the ovine genome has not been fully sequenced and the molecular tools available to study ovine brain injury are more limited than in rodents. The rodent models, including transgenic rodents, are ideal to provide more rapid, cost-effective access to cellular and molecular mechanisms, which can be validated subsequently in a large pre-clinical animal model, such as the instrumented fetal sheep.

The in utero nature of the instrumented fetal sheep preparation offers some practical advantages relative to other $e x$ utero models with a gyrencephalic brain (e.g., the piglet and nonhuman primate). To our knowledge, viable preterm fetal piglet models have not been achieved. Even in neonatal piglets, studies to date have only achieved relatively brief survival [30]. Instrumentation of the preterm nonhuman primate fetus carries a high risk for premature birth. Although controlled preterm delivery of the nonhuman primate fetus is feasible, neuropathological studies in preterm animals require ventilator support in an intensive care setting [31]. During prolonged ventilation, preterm baboons, for example, sustain disturbances in brain growth and development that make the interpretation of neuropathological studies more challenging [32-34]. A limitation of the instrumented fetal sheep preparation is that animals are not studied under ex utero conditions where the animals breathe room air.

Preterm ( 0.65 gestation or 95 days) fetal sheep models have multiple distinct advantages relative to small fetal and neonatal animals, which are limited by a lissencephalic brain that does not resemble the nascent gyrencephalic human cerebrum. In terms of its neurodevelopment, the immature ovine brain is similar to the preterm human brain between approximately 24 to 28 weeks in terms of the completion of neurogenesis, the onset of cerebral sulcation, and the detection of the cortical component of the auditory and somatosensory evoked potentials [35-38]. The long gestation of fetal sheep (145 days) allows selection of an appropriate developmental stage during which brain insults can be induced and evaluated.

The abundance of cerebral white matter and its anatomic similarities to that of the preterm infant make the fetal sheep ideal for neuropathological correlation with humans [39, 40], whereas rodents have a paucity of cerebral white matter 
that differs markedly from humans. White matter maturation in fetal sheep can be defined relative to humans through an assessment of oligodendrocyte lineage progression and myelination (Fig. 1). Oligodendrocyte development in the 0.65 gestation sheep fetus is similar to that of the 24- to 28week preterm human infant [41]. The late gestation ovine fetus ( 0.9 gestation; 135 days) displays oligodendrocyte development similar to the term human [42]. Thus, the investigator can choose the appropriate developmental timing for the insult to be evaluated. Although in both humans and sheep, myelination begins prenatally, a notable difference is that myelinogensis progresses more rapidly in sheep [41] than in humans [43], which continues to progress for months after term birth [44]. By contrast, the rodent displays accelerated white matter maturation and myelination that occurs entirely postnatally [45]. In neonatal rats, for example, the progression from human preterm equivalent to near-term equivalent oligodendrocyte maturation occurs rapidly during the first 5 days after birth [44].

The preterm fetal sheep is susceptible to acute [41] and chronic [46] WMI, which is very similar to humans in histopathological features. The fetal sheep cerebrum has a predilection for relatively selective WMI under conditions of moderate cerebral ischemia [41], whereas rodents have a propensity for mixed cerebral injury such that substantial gray

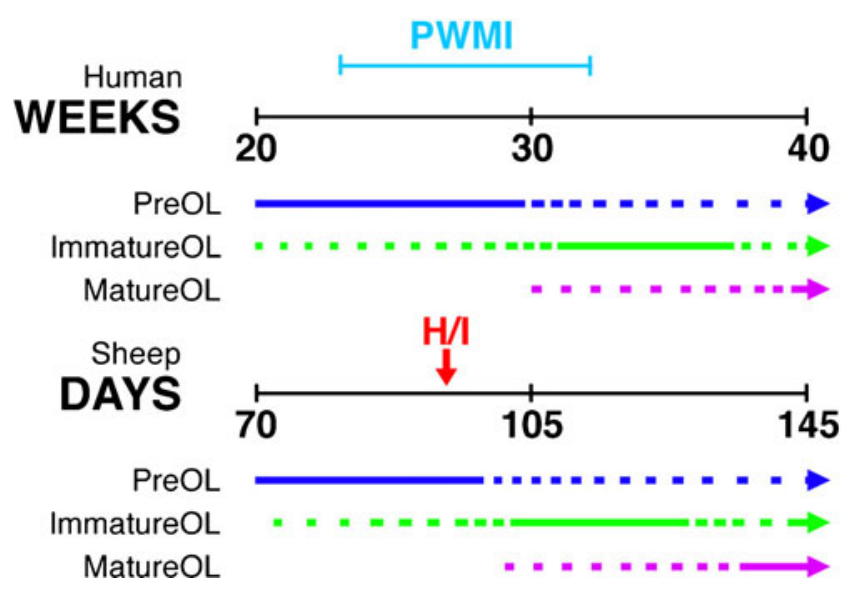

Fig. 1 Summary diagram that compares the timing of appearance of human versus ovine immature oligodendrocytes $(\mathrm{O} 4+\mathrm{O} 1+)$. The human is depicted during the latter half of gestation (20-40 weeks) and is based on data previously reported [133]. The progression of the oligodendrocyte lineage in fetal sheep white matter development has been previously described [41, 42]. The period shown ( 70-145 days) roughly corresponds with the fetal human during the latter half of gestation. Solid lines indicate the developmental period when each oligodendrocyte lineage stage predominates. The dotted lines indicate the period when these stages are a minor population. Note that in many studies hypoxia-ischemia (H/I) (arrow) is administered at 90 to 95 days, which coincides with the high-risk period for periventricular white matter injury (PWMI) at $\sim 23$ to 32 weeks. preOLs $=$ late oligodendrocyte progenitors matter injury accompanies WMI [47-52]. This shortcoming, for example, limits the relevance of rodent hypoxia-ischemia models for the study of myelination disturbances associated with chronic human WMI. Necrotic injury to cerebral gray matter contributes substantially to neuro-axonal degeneration as a cause of dysmyelination, which is not a prominent feature of WMI in either fetal sheep [53] or contemporary human cases of WMI [54]. The basis for this difference in susceptibility to gray matter injury in rodents relative to sheep and humans is likely to be multifactorial. The cerebrovascular supply of rodent white matter is structurally and physiologically very dissimilar to humans and sheep $[55,56]$. In addition, the timing of expression of the subtypes of glutamate receptors appears to differ between rodents and both humans and sheep [57-59], which may further contribute to the pronounced susceptibility of neonatal rodent cerebral gray matter to excitotoxic injury [60]. However, there are unexplained differences in glutamate transporter expression between sheep and humans during preterm white matter development where EAAT1 expression predominates in sheep [61], whereas EAAT2 expression predominates in humans [62].

The size of the preterm sheep fetus allows for chronic instrumentation to enable hemodynamic measurements, repeated access to blood and cerebrospinal fluid, and chronic electrophysiological recording of the fetal electroencephalogram [63]. In contrast to fetal sheep preparations, the small size of rodents is a major technical limitation for a wide range of invasive physiological measurements, as well as for studies that seek to achieve high resolution neuroimaging by using MRI $[46,64]$. Thus, it is feasible to study well-defined brain insults in fetal sheep with reliable measurements of blood pressure (BP), oxygenation, cerebral blood flow, and metabolism. Chronic instrumentation also allows a wide range of practical and clinically pertinent cerebral insults to be administered. These include global cephalic ischemia [65], systemic hypotension or hypoxemia [66-68], single or repeated cord occlusion [69-71], increased intracranial pressure [72], and administration of infectious agents or exogenous inflammatory mediators [73-75]. These insults can be graded in intensity and duration to mimic the human situation. In each case, the stressor can be well-described, if not regulated, by conventional monitoring measurements. Thus, a wide range of pathogenetic events can be evaluated physiologically and correlated neuropathologically with the distribution and extent of white matter damage. Moreover, the natural progression of various types of WMI can be evaluated in a time frame ranging from days to weeks to months after the insult. From a practical standpoint, the availability, cost, and ease of breeding the sheep makes it a more practical large animal model than the nonhuman primate. In addition, the size 
and docile nature of the sheep supports the feasibility of either in utero or ex vivo neuroimaging studies [46, 76].

\section{Hypoxia-Ischemia in Fetal Sheep Generates Pathological Features of WMI}

The ovine fetal sheep offers significant advantages to analyze systemic hemodynamic disturbances that regulate cerebral blood flow and metabolism in preterm cerebral white matter. The sheep fetus displays cerebral hemodynamics similar to humans and permits repeated physiological measurements in utero in the unanesthetized state. Importantly, similar to the human fetus [77-80], the fetal sheep displays a very limited range of cerebral autoregulation under normal conditions and a pressure-passive cerebral circulation when subjected to systemic hypoxia and associated hypotension [66, 81-84]. Moreover, measurements of BP, electroencephalography, blood oxygenation, and other vital variables can be correlated with acute changes in cerebral blood flow and metabolism.

Multiple lines of evidence support a role for cerebral ischemia in the pathogenesis of WMI in very low birth weight human infants $[5,85,86]$. Given the limitations of human studies to directly link blood flow disturbances with WMI, studies in fetal sheep have greatly strengthened our understanding of the contribution of cerebral hypoxiaischemia to WMI. These experimental studies support that a complex interplay of factors related to cerebrovascular immaturity predispose preterm cerebral white matter to injury from hypoxia-ischemia. Studies of global cerebral hypoperfusion found that the mid-gestation animal displayed a predilection to subcortical WMI, whereas the near term animal displayed predominantly parasagittal cortical neuronal injury [65, 87]. A variable degree of WMI was also detected after systemic hypotension arising from intermittent or partial umblical cord occlusion [88, 89]. By contrast, in the near term animal, repeated umbilical cord occlusion produced injury to both the periventricular white matter and the cerebral cortex [90]. Systemic hemorrhagic hypotension in the 0.75 gestation fetus resulted in mostly necrotic WMI with focal necrotic lesions or axonal swellings in the periventricular white matter [91]. Preterm ovine white matter lesions were also detected after repeated systemic fetal endotoxin exposure that triggered both transient hypoxemia and hypotension $[92,93]$. The importance of cerebral ischemia is supported by studies where WMI was detected only infrequently in models of hypoxemia in which a restriction in uteroplacental blood flow resulted in decreased oxygen delivery and mild acidemia to the fetus without systemic hypotension or cerebral hypoperfusion $[67,94,95]$. A model of fetal metabolic acidemia induced by maternal hypoxemia similarly produced mild-to-moderate injury in mid-gestation and nearterm sheep [96]. Hence, cerebral hypoperfusion in conjunction with hypoxia appears to be a critical factor to generate significant WMI in the preterm fetal sheep.

Fetal sheep have also provided an important model to define mechanisms of oxidative injury from cerebral hypoxiaischemia. In part, this is due to the large fetal cerebral hemispheres, which permit blood sampling from the venous sagittal sinus and placement of intracerebral probes for dialysis studies. Initial studies found that reperfusion after cerebral ischemia was an important source of free radical formation in the near term fetal sheep brain [97]. Partial umblical cord occlusion resulted in a delayed increase in lipid peroxidation in the frontal and parietal white matter in near term fetal sheep [98]. Dialysis studies in near term [99] and preterm [100, 101] sheep have demonstrated that the enhanced generation of reactive oxygen species does not occur during the immediate ischemiareperfusion period, but it is observed after a significant delay.

\section{Pathophysiological Mechanisms of WMI Related to the Brachiocephalic versus Carotid Occlusion Models of Global Cerebral Ischemia}

We have used 2 different approaches to achieve global cerebral ischemia. The first is to place an occluder on each of the carotid arteries in the neck. The second is to place a single occluder around the brachiocephalic artery in the chest. The relative advantages and disadvantages of each approach are discussed below. To confine the cerebral blood supply to the carotid arteries for both approaches, the occipito-vertebral anastomosis (OVA) are ligated bilaterally. These anastomoses connect the vertebral arteries, supplied by the thoracic aorta, with the external carotid arteries that are fed by the brachiocephalic [102].

The dominant factors related to the generation of WMI in most, if not all, models are: 1) the content of oxygen and glucose in the blood, 2) the driving or perfusion pressure and hence cerebral blood flow (CBF), and 3) the duration of the insult. In preterm fetal sheep, it is often feasible for these important variables to be manipulated and measured, either in groups of animals or in single individuals. The pathophysiological disturbances associated with these factors are variously weighted, depending on the model being used.

With models of maternal hypoxemia (e.g., high altitude or a low inspired oxygen fraction), fetal oxygenation falls while BP rises transiently, but then returns to normal, and then falls below normal $[67,68,96,103-106]$. Perinatal hypoxic-ischemic encepatholpathy models that involve the entire body frequently use umbilical cord occlusion and are accompanied by reduced systemic fetal arterial oxygen tension and content $[70,107,108]$. Injury in the umbilical cord occlusion models can be modulated by adjusting the duration of occlusion relative to the onset of systemic hypotension. In cord occlusion models, BP initially increases to compensate 
for diminished oxygenation, but as systemic and cardiac hypoxia progressively intensify, hemodynamic and cardiac decompensation occurs with a resultant fall in BP. It appears that significant brain damage occurs only when the hypoxia and hypotension are allowed to progress until near death conditions occur. However, as cardiovascular compensation fails with time, substantial albeit variable reductions in BP occur. The residual brain blood flow has seldom been measured, but for brain injury to occur, pressures must fall below one third of normal for at least 10 to 15 minutes, depending on the animal's age and the model being used [41, 109]. The importance of the central autonomic system and adrenal stress hormones remains unclear, as well as the systemic blood concentrations of metabolic substrates, such as glucose and products such as lactate. The fetal sheep brain, in particular when immature and hypoxemic, has essentially no ability to autoregulate [81-83]. Hence, in umbilical cord occlusion models, the fall in BP exacerbates the fetal brain hypoxia with a consequent reduction in $\mathrm{CBF}$ that leads to partial ischemia. Thus, occlusive cerebral ischemia models differ from systemic hypoxicischemic encepatholpathy models in several significant ways. A major practical advantage of the occlusive models is that the heart is largely unaffected. Essentially, cerebral damage can be reliably generated without the significant fetal deaths and morbidity that are inherently associated with other models that require near death conditions to ensure brain damage.

Unlike in utero systemic hypoxemia or asphyxia models, the models that use carotid or brachiocephalic artery (BCA) occlusions cause an immediate fall in perfusion pressure and $\mathrm{CBF}$, and thus have a well-defined onset of the insult $[41,65$, 110]. Cerebral ischemia in these models is generally global and severe but not complete, especially when collateral circulation is left intact (discussed as follows). Importantly, some CBF persists and the oxygenation and glucose levels of this residual flow have important influence on the extent of damage. The residual blood flow is the most difficult parameter to quantify. Even in fetal sheep models, CBF is only infrequently measured and BP is often used as a surrogate marker of $\mathrm{CBF}$.

There are a number of considerations that guide the selection of a model that uses carotid versus common BCA occlusion. However, the differences between bi-carotid and BCA occlusion are not as great as the difference between either of those models and cord occlusion models. The carotid arteries in the 0.65 sheep fetus are small and there is a relatively greater risk that commercially available occluders can inadvertently obstruct flow chronically if placement and routing techniques are not optimal. By contrast, the BCA is a larger and much more stable vessel for the placement of a single occluder [84, 103]. In sheep, the BCA supplies the entire head, including the carotids, as well as both axillary arteries, which supply the forelegs. The BCA is the only major artery that supplies the upper body. Occlusion of the BCA is similar to bilateral carotid occlusion in terms of perfusion to the head and brain, but different in that forelimb perfusion is also reduced, which does not occur if flow to both carotids is completely restricted.

One important consequence of the BCA occlusion model is that proximal $\mathrm{BP}$ to the rest of the fetal body and the placental circulation is subject to an elevated pressure. This probably results in an increase in umbilical flow that causes a moderate but significant rise in arterial oxygenation. The same effect probably occurs with bi-carotid occlusion, but it would be expected to be smaller. Either preparation can be coupled with a lowered maternal inspired oxygen fraction to counteract elevations in arterial oxygenation or even generate a fetal hypoxemia in addition to cerebral ischemia.

The OVA is small but potentially important in models where cephalic occlusions are studied $[65,102]$. They provide an important anastomosis between the anterior circulation provided by the carotid arteries and the posterior circulation derived from the vertebral arteries. The OVAs are likely to be variable in size from animal to animal, perhaps linked to variations in anatomy related to their supply at or near the Circle of Willis. They connect to the carotids near the lingual branch, and therefore they are distal to the BCA occluder, and to all but the most rostral placements of the carotid occluders. Hence, they act to support brain blood flow when systemic pressure is normal but BCA or carotid occlusions are used.

Hence, in both the bi-carotid and BCA occlusion models, it is important to ligate the OVAs to achieve near complete global cerebral ischemia. The exact amount of flow they provide, especially to the preterm fetus, has not been carefully determined. In normally oxygenated sheep, it is not clear whether there is a net flow and if there is, then in what direction. During either carotid or BCA occlusions, flow is certainly from the vertebrals to the carotids distal to the site of placement of the occluders. The amount of flow in the OVA, while likely to be variable from animal to animal, is clearly sufficient to cause variability in brain damage in the cephalic ischemia models, unless they are ligated.

\section{Role of Vascular End Zones in Cerebral WMI}

The role of cerebral vascular immaturity in the pathogenesis of WMI has been difficult to study in preterm infants. Analysis of the vascular supply to the periventricular white matter has yielded controversial results. The periventricular white matter has 2 major blood supplies. Perforating arteries branch from leptomeningeal arteries, penetrate the cerebral cortex, and terminate as capillary beds adjacent to the ventricles. Branches of choroidal and striate arteries project toward the lateral ventricles and then deviate away from the ventricle toward their final termination in vascular capillary beds in the periventricular white matter. Although these vascular beds may collectively form vascular end zones and border zones that render the periventricular white matter 
particularly susceptible to ischemia, physiological studies in support of this concept are lacking. Hence, existence of these border zones remains controversial [111-113]. The presence of these vascular zones would provide a mechanism for WMI based on the notion that when periventricular white matter flow falls below a critical threshold, this region would display greater susceptibility to WMI relative to the better-perfused cerebral cortex.

To address this controvery, we developed methods to spatially quantify $\mathrm{CBF}$, because large differences in flow in small regions can be obscured when averaged with larger unaffected regions, as is the case with more global measures of cerebral blood flow [41]. To be able to analyze CBF disturbances in regions of periventricular white matter vulnerable to hypoxicischemic injury, we developed a novel method to achieve high resolution spatial blood flow measurements on the brains of immature fetal sheep. This was achieved by determining the location in the fetal brain of thousands of individual fluorescently labeled microspheres [41, 109]. This high resolution blood flow technique is illustrated in Fig. 2. Basal or control blood flow in 2 of 16 "virtual" 2-mm thick coronal sections of a 0.65 gestation fetal sheep brain are shown. The image in the upper left represents a three-dimensional reconstruction of the approximately 1000 Imaging CryoMicrotome images (Barlow Scientific, Olympia, WA) obtained to define the location of each microsphere. Two coronal two-dimensional virtual sections, at the level of the (image 1) parietal and (image 2) frontal white matter (Fig. 2), where white matter damage was observed, show the two-dimensional representation of blood flow under basal conditions. Blood flow is displayed as a "convolved" image of the density of microsphere distribution and quantified $(\mathrm{ml} / \mathrm{min})$ in the pseudocolor scale shown. Basal blood flow was particularly high, for example, in the thalamic nuclear groups in the midbrain (Fig. 2, image 1, arrow) relative to the parietal cortex. By contrast, the basal blood flow of the parietal and frontal periventricular white matter was markedly lower (Fig. 2, arrowheads). Cortical blood flow is much higher than periventricular white matter flow. This degree of spatial resolution can only be achieved in brains at least the size of the immature fetal sheep.

To seek evidence for vascular end and border zones in fetal cerebral white matter, we measured blood flow in histopathologically defined regions of injury in cerebral cortex and white matter in preterm fetal sheep [109]. Although white matter blood flow was lower than cerebral gray matter, there was no evidence for pathologically significant gradients of fetal blood flow within the periventricular white matter under conditions of global partial ischemia or reperfusion. White matter lesions did not localize to regions susceptible to greater ischemia, nor did less vulnerable regions of cerebral white matter have greater flow during ischemia. An alternative explanation for the topography of cerebral white matter lesions is the distribution of susceptible cell types (described as follows), particularly late oligodendrocyte (OLs) progenitors (preOLs) that are particularly susceptible to hypoxia-ischemia.

\section{Relative Contributions of Hypoxia-Ischemia and OL Lineage Immaturity to Acute WMI}

The preterm fetal sheep ( 0.65 gestation) displays heterogeneous OL lineage maturation in frontal periventricular white matter [41], which allowed us to define the relative contributions of oligodendroglial maturational factors and vascular factors to acute WMI. OL lineage maturation in medial periventricular white matter (PVWM) was similar to that of a human $(\sim 23-$
Fig. 2 Quantification of fetal cerebral blood flow in situ under conditions of basal flow. The top image represents a three-dimensional surface reconstruction (Volocity, Improvision, Lexington, MA) of fluorescence images of a 0.65 gestation ovine control brain that indicates the frontal and parietal levels to which the lower blood flow images correspond. Representative pseudocolor basal blood flow images show higher blood flow (arrows) in the pons (image 1) and subcortical gray matter (image 2), and lower flow (dark blue) in the periventricular white matter (PVWM) (arrowheads)

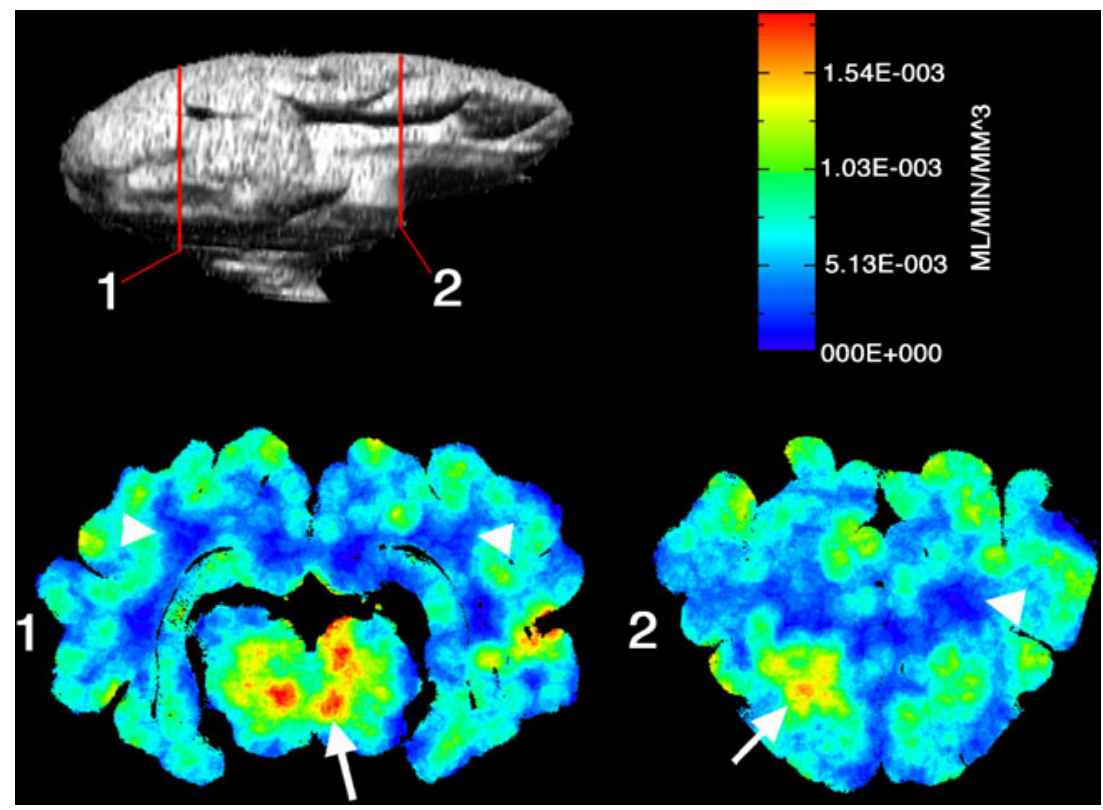


28 weeks gestation) in that preOLs were the major OL stage present. By contrast, lateral PVWM was more differentiated and contained predominantly pre-myelinating and early myelinating immature OLs. Surprisingly, we found that moderate cerebral ischemia did not uniformly damage the PVWM. The medial and lateral PVWM sustained differing degrees of acute injury, even though they sustained a similar degree of low flow during prolonged ischemia-reperfusion. Hence, while global ischemia was necessary for WMI, no regional differences in blood flow were found within the PVWM under basal or ischemic conditions to account for the differences in cell death between medial and lateral PVWM. Rather, differences in the topography of WMI were closely correlated with the distribution of vulnerable preOLs. Interestingly, in regions of preOL degeneration, other neural cell types (astrocytes, microglia, and axons) were markedly more resistant to injury.

In a fetal rabbit model of placental insufficiency, significant global fetal hypoxia-ischemia caused minimal WMI at fetal day 22, but a similar insult 3 days later in gestation caused pronounced WMI [114]. The relative susceptibility of the white matter at these 2 developmental ages coincided with the timing of appearance of susceptible preOLs. Taken together, these findings suggest that perturbations in cerebral blood flow are necessary, but not sufficient to explain the distribution of WMI. The developmental predilection for WMI appears to be related to both the timing of appearance and regional distribution of susceptible preOLs.

\section{Role of High-Field MRI to Define Pathological Features of WMI}

Although MRI is the optimal imaging modality to define WMI in preterm survivors [115-118], the histopathological features of MRI signal abnormalities have been mostly defined for WMI where periventricular leukomalacia predominates [64, 119-123]. With the pronounced shift to milder forms of human WMI defined by quantitative and diffusionweighted MRI, there is a need to define the cellular features of these lesions. We have recently analyzed diffuse lesions in a preterm fetal sheep model where animals survived for 1 or 2 weeks after global cerebral ischemia. This preparation generated a spectrum of WMI very similar to that observed from human autopsy studies, as well as a reduction in cerebral WM volume similar to that observed in preterm survivors [116-118]. We developed registration algorithms to analyze the histopathological features of 3 classes of MRI-defined lesions identified by ex vivo imaging at high field (12 Tesla) [46]. Each lesion type displayed unique astroglial and microglial responses that corresponded to distinct forms of necrotic or non-necrotic WMI.

The major type of lesion identied by MRI was a novel diffuse hypointense signal abnormality (D-hypo) identified on T2-weighted images at 1 and 2 weeks after global cerebral ischemia (Fig. 3A). The physical-chemical basis for these highly hypointense lesions is unclear, but they did not correspond to hemorrhage. These lesions comprised $89 \%$ of total lesion volume at 1 week and corresponded to histopathogically defined lesions that were highly enriched in reactive astrocytes, but were only modestly enriched in activated microglia at 1 or 2 weeks (Fig. 3B, C). This diffuse hypointense signal abnormality, thus appears to be a unique MRI signature for diffuse gliotic lesions dominated by reactive astrogliosis. Importantly, large lesions, which comprised $97 \%$ of the WMI identified, were detected with a sensivity of $100 \%$ at 1 week and $75 \%$ at 2 weeks.

With high-field MRI, we also identifed a second type of novel focal hypointense signal abnormality (F-hypo) on T2weighted images at 2 weeks after global cerebral ischemia (Fig. 4A). Although these lesions were observed in $50 \%$ of
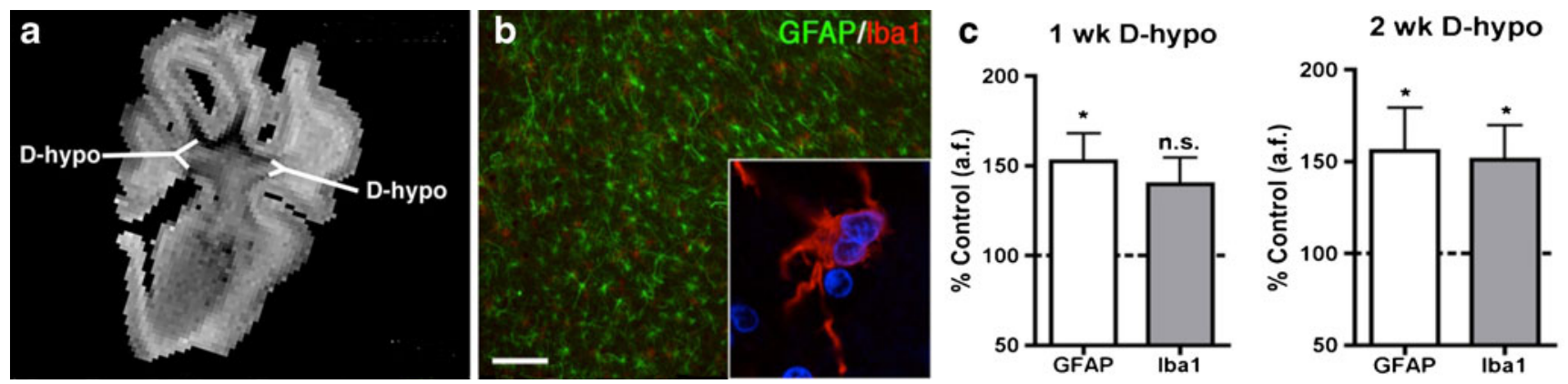

Fig. 3 Progressive histopathological and high-field magnetic resonance image (MRI)-defined features of diffuse cerebral white matter gliosis in chronic lesions generated in the 0.65 gestation ovine brain at 1 or 2 weeks after global cerebral ischemia. (A) Representative appearance and distribution of diffuse hypointense (D-hypo) lesions seen on a $T_{2} \mathrm{~W}$ image at 1 week after injury. (B) Diffuse white matter injury (WMI) had pronounced astrogliosis defined by immunohistochemical staining of reactive astrocytes with glial fibrillary acidic protein
(GFAP) (green) and a lesser population of Iba1-labeled microglia/ macrophages (red) with a reactive morphology (inset). Nuclei in the inset are visualized with Hoechst 33342 (blue). Bar=100 $\mu \mathrm{m}$. (C) Quantification of GFAP-labeled astrocytes and Iba1-labeled microglia within MRI-defined white matter signal abnormalities at 1 and 2 weeks after global ischemia. The D-hypo lesions had significantly elevated GFAP, consistent with a diffuse astrogliotic response to injury. ${ }^{*} p<$ $0.05 ;$ n.s. $=$ not significant 

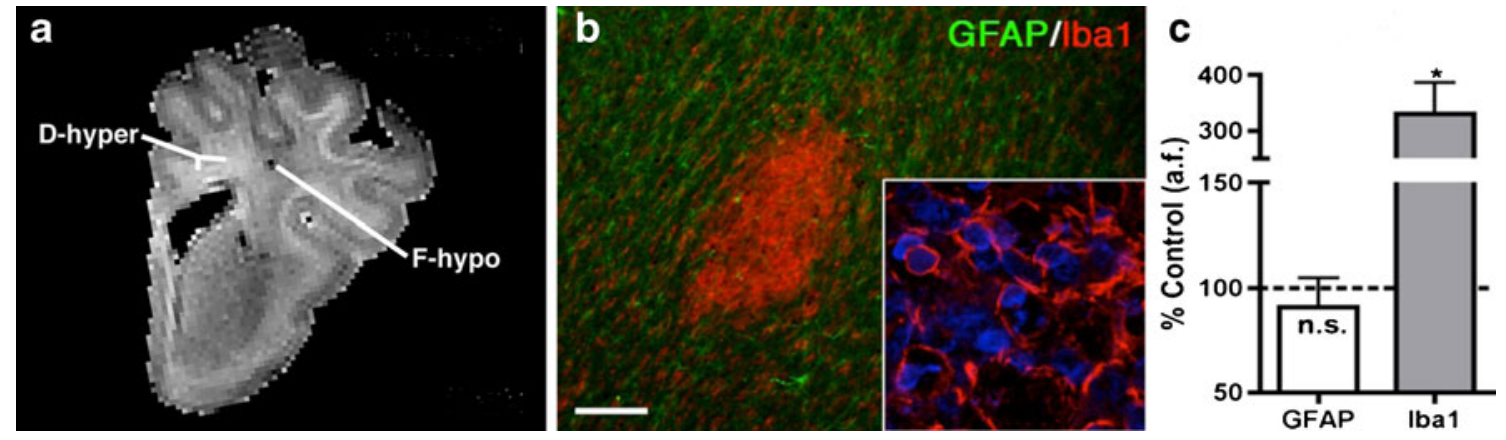

Fig. 4 Histopathological and high-field magnetic resonance image (MRI)-defined features of microscopic necrosis detected in fetal ovine cerebral white matter at 2 weeks after global cerebral ischemia. (A) Representative appearance of a focal hypointense (F-hypo) lesion seen on a $\mathrm{T}_{2} \mathrm{~W}$ image at 2 weeks after injury. Note the substantial difference in the F-hypo lesion relative to a diffuse gliotic lesion at 2 weeks, which appears more hyperintense (D-hyper). (B) A typical microscopic

the animals, they only comprised $\sim 1.5 \%$ of total lesion volume and corresponded to histopathogically defined lesions that were highly enriched in reactive microglia and macrophages, but contained a paucity of astrocytes (Fig. 3B, C). Thus, this focal hypointense signal abnormality corresponded to small discrete regions of focal microscopic necrosis that were detected at submillimeter resolution. Both the incidence and lesion burden of these small necrotic foci of WMI were quite similar to that observed in human WMI [54]. Although these lesions are readily detected by histopathology, they are not readily detected by MRI at lower field strength. Hence, high-field MRI provides a unique signature for microscopic necrosis.

A third class of lesions detected on T2-weighted images by high-field MRI were focal hyperintense lesions (F-hyper) (Fig. 5A). These lesions also had low values for fractional anisotropy and high apparent diffusion coefficient values, consistent with lesions with high water content. In fact, the F-hyper lesions were defined histologically by a progressive loss of astroyctes between 1 and 2 weeks and a marked enrichment necrotic lesion defined by a discrete focus of immunohistochemical staining for reactive microglia and macrophages with Iba1 (red; inset) and a paucity of staining for astrocytes with glial fibrillary acidic protein (GFAP) (green). Nuclei in the inset are visualized with Hoechst 33342 (blue). Bar=100 $\mu \mathrm{m}$. (C) F-hypo lesions had markedly increased Ibal labeling and no significant difference in GFAP labeling versus control. ${ }^{*} p<0.05 ;$ n.s. $=$ not significant

in reactive micrglia and macrophages (Fig. 5B, C) and features of acute axonal degeneration, including dystrophic axons and axonal spheroids (not shown). Thus, these lesions displayed the characteristic features of a diffuse macroscopic necrotic lesion.

These ex vivo high-field MRI studies suggest that current clinical MRI field strength may be a limiting factor to detect diffuse gliosis, as well as microscopic necrosis (discussed as follows). Additional clinical pathological studies in fetal sheep or other relevant models are needed to determine whether highfield MRI can provide greater sensitivity to identify diffuse WMI than what is currently feasible at lower field strengths.

\section{MRI-Guided Ultrastructural Studies of Axonal Degeneration in WMI}

Axonal degeneration is a common feature of necrotic WMI and can be detected both within and distal to large necrotic foci [124]. Whether primary axonal injury occurs in cases of
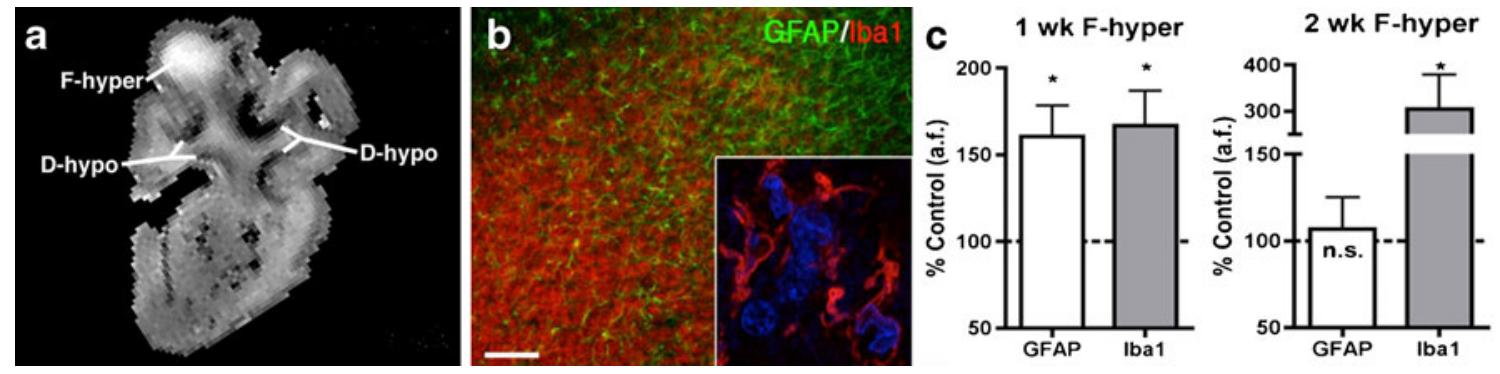

Fig. 5 Histopathological and high-field magnetic resonance image (MRI)-defined features of diffuse macroscopic necrosis detected in fetal ovine cerebral white matter at 1 and 2 weeks after global cerebral ischemia. (A) Representative appearance from the largest focal hyperintense (F-hyper) lesion seen on a $\mathrm{T}_{2} \mathrm{~W}$ image at 1 week after injury. These lesions typically localized to subcortical white matter. Note the substantial difference in the F-hyper lesion relative to the diffuse gliotic lesions, which appears much more hypointense (D-hypo). (B) A typical macroscopic necrotic lesion defined by diffuse dense staining for reactive microglia and macrophages with Ibal (red; inset) and a paucity of GFAP-labeled astrocytes. Nuclei in the inset are visualized with Hoechst 33342 (blue). Bar=100 $\mu \mathrm{m}$. (C) F-hyper lesions displayed a progressive decrease in glial fibrillary acidic protein (GFAP) staining and markedly increased Iba1 labeling for microglia by 2 weeks after global ischemia. ${ }^{*} p<0.05 ;$ n.s. $=$ not significant 
diffuse WMI that lack significant necrosis has received limited study. During the acute phase of WMI, after global hypoxia-ischemia, fetal sheep that lacked necrotic WMI did not show evidence of axonal degeneration [41]. We used quantitative electron microscopy studies in our preterm fetal sheep model of global cerebral ischemia to define the extent of axonal degeneration in MRI-defined diffuse WMI [53]. During the chronic phase of WMI from this same fetal sheep preparation [46] and human [54], microscopic foci of necrosis were observed that were rich in microglia, but lacked astrocytes or axons. However, no significant axonal degeneration, axonal loss or shift in the distribution of axon calibers was observed by quantitative electron microscopy studies in preterm fetal sheep [53]. Hence, the contribution of microscopic necrosis to axonal loss with secondary myelination failure appears to be low, but there is a need for further human neuropathological studies with sensitive markers of axonal injury that are applied to cases of diffuse WMI that lack necrosis.

\section{Final Conclusions}

The preterm human infant displays unique patterns of cerebral injury that can now be closely replicated in the preterm fetal sheep. Despite the higher costs and technical challenges of preterm fetal sheep models, they provide powerful access to pre-clinical questions related to the pathophysiology of WMI. Recent advances include spatially defined measurements of cerebral blood flow in utero, the definition of cellular maturational factors that define the topography of WMI, and the application of high-field neuroimaging to define MRI signatures for specific types of chronic WMI. There is a critical need to further define the cellular and molecular mechanisms that mediate the progression of cerebral white and gray matter injury. Such information is important for the rationale design of therapies targeted to block the initial or secondary phases of injury, and to promote regeneration and repair during the chronic phase.

With few exceptions, most studies have focused on models of isolated exposure to cerebral hypoxia-ischemia or maternal fetal infection. Future models are needed that more closely reproduce the spectrum of insults that appear to contribute to cerebral injury in human preterm infants. For example, there are potential limitations of studies of healthy animals that do not fully address the impact of clinically common complications, which influence the response to a single episode of hypoxia-ischemia. These include the influence of antecedent recurrent transient or prolonged hypoxia-ischemia, placental insufficiency, or chronic in utero ischemia. Depending on the timing of the insult, fetal infection has the potential to either exacerbate ischemic injury or protect against it via mechanisms that may involve ischemic tolerance. Elegant studies in neonatal rodents have not been sufficiently replicated in large preterm models [125-127]. To date there have been virtually no neurobehavioral studies of preterm fetal sheep that have sustained WMI. Greater clinical relevance of fetal sheep models of WMI also may be achieved by addressing common confounders of neonatal care that include the influence of nutritional status, stressors, painful exposures, and recurrent sedative and anesthetic exposure [128-132]. All are likely to adversely influence neonatal brain development and the impact of WMI on subsequent long-term motor and cognitive development via mechanisms that are currently not well understood.

Acknowledgments Supported by the National Institutes of Neurological Diseases and Stroke (1RO1NS054044, R37NS045737-06S1/ $06 \mathrm{~S} 2$ to $\mathrm{SAB}$ and $1 \mathrm{~F} 30 \mathrm{NS} 066704$ to $\mathrm{AR}$ ); the National Institute of Child Health and Human Development (NICHD P01HD34430); the American Heart Association (to SAB); and the March of Dimes Birth Defects Foundation (to SAB).

Required Author Forms Disclosure forms provided by the authors are available with the online version of this article.

\section{References}

1. Ferriero DM. Can we define the pathogenesis of human periventricular white-matter injury using animal models? J Child Neurol 2006;21:580-581.

2. Gressens P, Le Verche V, Fraser M, et al. Pitfalls in the quest of neuroprotectants for the perinatal brain. Dev Neurosci 2011;33:189198.

3. Gunn AJ, Battin M, Gluckman PD, Gunn TR, Bennet L. Therapeutic hypothermia: from lab to NICU. J Perinat Med 2005;33:340-346.

4. Higgins RD, Raju T, Edwards AD, et al. Hypothermia and other treatment options for neonatal encephalopathy: an executive summary of the Eunice Kennedy Shriver NICHD workshop. J Pediatr 2011;159:851-858.

5. Volpe JJ. Neurology of the Newborn. Philadelphia: W.B. Saunders, 2008.

6. Back S. Mechanisms of Acute and Chronic Brain Injury in the Preterm Infant. In: Miller S, Shevell M, eds. Acquired Brain Injury. London: Mac Keith Press, 2012.

7. Wilson-Costello D, Fridedman H, Minich N, Fanaroff A, Hack M. Improved survival rates with increased neurodevelopmental disability for extremely low birth weight infants in the 1990s. Pediatrics 2005;115:997-1003.

8. Hack M, Taylor H, Drotar D, et al. Chronic conditions, functional limitations, and special health care needs of school-aged children born with extremely low-birth-weight in the 1990's. JAMA 2005;294:318-325.

9. Miller SP, Ferriero DM, Leonard C, et al. Early brain injury in premature newborns detected with magnetic resonance imaging is associated with adverse neurodevelopmental outcome. J Pediatr 2005;147:609-616.

10. Beaino G, Khoshnood B, Kaminski M, et al. Predictors of cerebral palsy in very preterm infants: the EPIPAGE prospective population-based cohort study. Dev Med Child Neurol 2010;52: e119-e125.

11. Mercier CE, Dunn MS, Ferrelli KR, Howard DB, Soll RF. Neurodevelopmental outcome of extremely low birth weight infants 
from the Vermont Oxford network: 1998-2003. Neonatology 2010;97:329-338.

12. Liu J, Li J, Qin GL, Chen YH, Wang Q. Periventricular leukomalacia in premature infants in mainland China. Am J Perinatol 2008;25:535-540.

13. Spittle AJ, Cheong J, Doyle LW, et al. Neonatal white matter abnormality predicts childhood motor impairment in very preterm children. Dev Med Child Neurol 2011;53:1000-1006.

14. Litt J, Taylor H, Klein N, Hack M. Learning disabilities in children with very low birthweight:prevalence, neuropsychological correlates and educational interventions. J Learn Disabil 2005;8:130-141.

15. Jacobson LK, Dutton GN. Periventricular leukomalacia: an important cause of visual and ocular motility dysfunction in children. Surv Ophthalmol 2000;45:1-13.

16. Glass HC, Fujimoto S, Ceppi-Cozzio C, et al. White-matter injury is associated with impaired gaze in premature infants. Pediatr Neurol 2008;38:10-15.

17. Soria-Pastor S, Gimenez M, Narberhaus A, et al. Patterns of cerebral white matter damage and cognitive impairment in adolescents born very preterm. Int J Dev Neurosci 2008;26:647-654.

18. Anderson PJ, De Luca CR, Hutchinson E, Spencer-Smith MM, Roberts G, Doyle LW. Attention problems in a representative sample of extremely preterm/extremely low birth weight children. Dev Neuropsychol 2011;36:57-73.

19. Constantinou JC, Adamson-Macedo EN, Mirmiran M, Fleisher BE. Movement, imaging and neurobehavioral assessment as predictors of cerebral palsy in preterm infants. J Perinatol 2007;27:225-229.

20. Spittle AJ, Brown NC, Doyle LW, et al. Quality of general movements is related to white matter pathology in very preterm infants. Pediatrics 2008;121(5):e1184-e1189.

21. Spittle AJ, Boyd RN, Inder TE, Doyle LW. Predicting motor development in very preterm infants at 12 months' corrected age: the role of qualitative magnetic resonance imaging and general movements assessments. Pediatrics 2009;123:512-517.

22. Bax M, Tydeman C, Flodmark O. Clinical and MRI correlates of cerebral palsy: the European Cerebral Palsy Study. JAMA 2006;296:1602-1608.

23. Pagliano E, Fedrizzi E, Erbetta A, et al. Cognitive profiles and visuoperceptual abilities in preterm and term spastic diplegic children with periventricular leukomalacia. J Child Neurol 2007;22:282-288.

24. Li AM, Chau V, Poskitt KJ, et al. White matter injury in term newborns with neonatal encephalopathy. Pediatr Res 2009;65:85-89.

25. Lasry O, Shevell MI, Dagenais L. Cross-sectional comparison of periventricular leukomalacia in preterm and term children. Neurology 2010;74:1386-1391.

26. Wernovsky G, Shillingford A, Gaynor J. Central nervous system outcomes in children with complex congenital heart disease. Curr Opin Cardiol 2005;20:94-99.

27. Miller S, McQuillen P, Hamrick S, et al. Abnormal brain development in newborns with congenital heart disease. N Engl J Med 2007;357:1971-1973.

28. Licht DJ, Shera DM, Clancy RR, et al. Brain maturation is delayed in infants with complex congenital heart defects. J Thorac Cardiovasc Surg 2009;137:529-537.

29. Centers for Disease Control and Prevention. Economic costs associated with mental retardation, cerebral palsy, hearing loss and vision impairment — United States, 2003. MMWR Morb Mortal Wkly Rep 2004;53:57-59.

30. Thoresen M, Haaland K, Loberg EM, et al. A piglet survival model of posthypoxic encephalopathy. Pediatr Res 1996;40:738-748.

31. Griffith JL, Shimony JS, Cousins SA, et al. MR imaging correlates of white-matter pathology in a preterm baboon model. Pediatr Res 2012;71:185-191.

32. Loeliger $\mathrm{M}$, Inder $\mathrm{T}$, Cain $\mathrm{S}$, et al. Cerebral outcomes in a preterm baboon model of early versus delayed nasal continuous positive airway pressure. Pediatrics 2006;118:1640-1653.
33. Loeliger M, Inder TE, Shields A, et al. High-frequency oscillatory ventilation is not associated with increased risk of neuropathology compared with positive pressure ventilation: a preterm primate model. Pediatr Res 2009;66:545-550.

34. Verney C, Rees S, Biran V, Thompson M, Inder T, Gressens P. Neuronal damage in the preterm baboon: impact of the mode of ventilatory support. J Neuropathol Exp Neurol 2010;69:473-482.

35. Barlow R. The foetal sheep: morphogenesis of the nervous system and histochemical aspects of myelination. J Comp Neurol 1969;135:249-262.

36. Bernhared C, Kolmodin G, Meyerson B. On the prenatal development of function and structure in the somesthetic cortex of the sheep. Prog Brain Res 1967;2:60-77.

37. Cook C, Gluckman P, Johnston B, Williams C. The development of the somatosensory evoked potential in the unanaesthetized fetal lamb. J Dev Physiol 1987;9:441-456.

38. Cook C, Williams C, Gluckman P. Brainstem auditory evoked potential in the fetal lamb, in utero. J Dev Physiol 1987;9:429-440.

39. Gluckman P, Parsons Y. Stereotaxic method and atlas for the ovine fetal forebrain. J Dev Physiol 1983;5:101-128.

40. Vanderwolf C, Cooley R. The sheep brain: a photographic series, 2nd ed. London, Ontario, Canada: A. J. Kirby Co., 1990.

41. Riddle A, Luo N, Manese M, et al. Spatial heterogeneity in oligodendrocyte lineage maturation and not cerebral blood flow predicts fetal ovine periventricular white matter injury. J Neurosci 2006;26:3045-3055.

42. Back SA, Riddle A, Hohimer AR. Role of instrumented fetal sheep preparations in defining the pathogenesis of human periventricular white matter injury. J Child Neurol 2006;21:582589.

43. Back SA, Luo NL, Borenstein NS, Volpe JJ, Kinney HC. Arrested oligodendrocyte lineage progression during human cerebral white matter development: dissociation between the timing of progenitor differentiation and myelinogenesis. J Neuropathol Exp Neurol 2002;61:197-211.

44. Dean J, Moravec M, Grafe M, et al. Strain-specific differences in perinatal rodent oligodendrocyte lineage progression and its correlation with human. Dev Neurosci 2011;33:251-260.

45. Craig A, Luo NL, Beardsley DJ, et al. Quantitative analysis of perinatal rodent oligodendrocyte lineage progression and its correlation with human. Exp Neurol 2003;181:231-240.

46. Riddle A, Dean J, JR B, et al. Histopathological correlates of MRI-defined chronic perinatal white matter injury. Ann Neurol 2011;70:493-507.

47. Segovia K, Mcclure M, Moravec M, et al. Arrested oligodendrocyte lineage maturation in chronic perinatal white matter injury. Ann Neurol 2008;63:517-526.

48. Back SA, Han BH, Luo NL, et al. Selective vulnerability of late oligodendrocyte progenitors to hypoxia-ischemia. J Neurosci 2002;22:455-463.

49. Follet PL, Rosenberg PA, Volpe JJ, Jensen FE. NBQX attenuates excitotoxic injury to the developing white matter. J Neurosci 2000;20:9235-9241.

50. Uehara H, Yoshioka H, Kawase S, et al. A new model of white matter injury in neonatal rats with bilateral carotid artery occlusion. Brain Res 1999;837:213-220.

51. Olivier P, Baud O, Evrard P, Gressens P, Verney C. Prenatal ischemia and white matter damage in rats. J Neuropathol Exp Neurol 2005;64:9d98-1006.

52. Marret S, Mukendi R, Gadisseux J-F, Gressens P, Evrard P. Effect of ibotenate on brain development: an excitotoxic mouse model of microgyria and posthypoxic-like lesions. J Neuropathol Exp Neurol 1995;54:358-370.

53. Riddle A, Maire J, Gong X, et al. Differential susceptibility to axonopathy in necrotic and non-necrotic perinatal white matter injury. Stroke 2012;43:178-184. 
54. Buser J, Maire J, Riddle A, et al. Arrested pre-Oligodendrocyte maturation contributes to myelination failure in premature infants. Ann Neurol 2012;71:93-109.

55. Vannucci RC, Lyons DT, Vasta F. Regional cerebral blood flow during hypoxia-ischemia in immature rats. Stroke 1988;19:245250.

56. Vannucci RC, Vannucci SJ. Perinatal hypoxic-ischemic brain damage: evolution of an animal model. Dev Neurosci 2005;27:81-86.

57. Talos D, Fishman R, Park H, et al. Developmental regulation of alpha-amino-3-hydroxy-5-methyl-4-isoxazole-propionic acid receptor subunit expression in forebrain and relationship to regional susceptibility to hypoxic/ischemic injury. I. Rodent cerebral white matter and cortex. J Comp Neurol 2006;497:42-60.

58. Talos DM, Follett PL, Folkerth RD, et al. Developmental regulation of alpha-amino-3-hydroxy-5-methyl-4-isoxazole-propionic acid receptor subunit expression in forebrain and relationship to regional susceptibility to hypoxic/ischemic injury. II. Human cerebral white matter and cortex. J Comp Neurol 2006;497:6177.

59. Dean JM, Fraser M, Shelling AN, et al. Ontogeny of AMPA and NMDA receptor gene expression in the developing sheep white matter and cerebral cortex. Brain Res Mol Brain Res 2005;139:242-250.

60. Johnston MV. Excitotoxicity in neonatal hypoxia. MRDD Res Rev 2001;7:229-234.

61. Northington FJ, Traystman RJ, Koehler RC, Martin LJ. GLT1, glial glutamate transporter, is transiently expressed in neurons and develops astrocyte specificity only after midgestation in the ovine fetal brain. J Neurobiol 1999;39:515-526.

62. Desilva TM, Kinney HC, Borenstein NS, et al. The glutamate transporter EAAT2 is transiently expressed in developing human cerebral white matter. J Comp Neurol 2007;501:879-890.

63. Gunn AJ, Bennet L. Fetal hypoxia insults and patterns of brain injury: insights from animal models. Clin Perinatol 2009;36:579593.

64. Lodygensky G, West T, Moravec M, et al. Diffusion characteristics associated with neuronal injury and glial activation following hypoxia-ischemia in the immature brain. Magn Reson Med 2011;66:839-835.

65. Reddy K, Mallard C, Guan J, et al. Maturational change in the cortical response to hypoperfusion injury in the fetal sheep. Pediatr Res 1998;43:674-682.

66. Szymonowicz W, Walker A, Yu V, Stewart M, Cannata J, Cussen L. Regional cerebral blood flow after hemorrhagic hypotension in the preterm, near-term, and newborn lamb. Pediatr Res 1990;28:361-366.

67. Rees S, Breen S, Loeliger M, McCrabb G, Harding R. Hypoxemia near mid-gestation has long-term effects on fetal brain development. J Neuropathol Exp Neurol 1999;58:932-945.

68. Gleason CA, Hamm C, Jones MD Jr. Effect of acute hypoxemia on brain blood flow and oxygen metabolism in immature fetal sheep. Am J Physiol 1990;258(4 pt 2):H1064-H1069.

69. Fadlkowski A, Hammond R, Han V, Richardson B. Apoptosis in the preterm and near term ovine fetal brain and the effect of intermittent umbilical cord occlusion. Dev Brain Res 2002;136:165-173.

70. Bennet L, Rossenrode S, Gunning MI, Gluckman PD, Gunn AJ. The cardiovascular and cerebrovascular responses of the immature fetal sheep to acute umbilical cord occlusion. J Physiol $1999 ; 517$ (pt 1):247-257.

71. Welin AK, Svedin P, Lapatto R, et al. Melatonin reduces inflammation and cell death in white matter in the mid-gestation fetal sheep following umbilical cord occlusion. Pediatr Res 2007;61:153-158.

72. Harris AP, Koehler RC, Gleason CA, Jones MD Jr., Traystman RJ. Cerebral and peripheral circulatory responses to intracranial hypertension in fetal sheep. Circ Res 1989;64:991-1000.
73. Rees S, Hale N, De Matteo R, et al. Erythropoietin is neuroprotective in a preterm ovine model of endotoxin-induced brain injury. J Neuropathol Exp Neurol 2010;69:306-319.

74. Duncan J, Cock M, Scheerlinck J, et al. White matter injury after repeated endotoxin exposure in the preterm ovine fetus. Ped Res 2006;52:941-949.

75. Dean J, van de Looij Y, Sizonenko S, et al. Delayed cortical impairment following lipopolysaccharide exposure in preterm fetal sheep. Ann Neurol 2011;70:846-856.

76. Sorensen A, Pedersen M, Tietze A, Ottosen L, Duus L, Uldbjerg $\mathrm{N}$. BOLD MRI in sheep fetuses: a non-invasive method for measuring changes in tissue oxygenation. Ultrasound Obstet Gynecol 2009;34:687-692.

77. Pyrds O. Control of cerebral circulation in the high-risk neonate. Ann Neurol 1991;30:321-329.

78. Menke J, Michel E, Hildebrand S. Cross-spectral analysis of cerebral autoregulation dynamics in high risk preterm infants during the perinatal period. Pediatr Res 1997;42:690-699.

79. du Plessis A. Cerebrovascular injury in premature infants: current understanding and challenges for future prevention. Clin Perinatol 2008;35:609-641.

80. Soul J, Hammer P, Tsuji M, et al. Fluctuating pressure-passivity is common in the cerebral circulation of sick premature infants. Pediatr Res 2007;61:467-473.

81. Papile L, Rudolph AM, Heymann M. Autoregulation of cerebral blood flow in the preterm fetal lamb. Pediatr Res 1985;19:159-161.

82. Tweed W, Cote J, Pash M, Lou H. Arterial oxygenation determines autoregulation of cerebral blood flow in fetal lamb. Pediatr Res 1985;17:246-249.

83. Helou S, Koehler RC, Gleason CA, Jones MD, Traystman RJ. Cerebrovascular Autoregulation During Fetal Development in Sheep. Am J Physiol 1994;266:H1069-H1074.

84. Hohimer AR, Bissonnette JM. Effects of cephalic hypotension, hypertension, and barbiturates on fetal cerebral flood flow and metabolism. Am J Obstet Gynecol 1989;161:1344-1351.

85. Greisen G. To autoregulate or not to autoregulate - that is no longer the question. Semin Pediatr Neurol 2009;16:207-215.

86. Tsuji M, Saul J, du Plessis A, et al. Cerebral intravascular oxygenation correlates with mean arterial pressure in critically ill premature infants. Pediatrics 2000;106:625-632.

87. Raad RA, Tan WK, Bennet L, et al. Role of the cerebrovascular and metabolic responses in the delayed phases of injury after transient cerebral ischemia in fetal sheep. Stroke 1999;30:2735-2741.

88. Clapp III J, Peress N, Wesley M, Mann L. Brain damage after intermittent partial cord occlusion in the chronically instrumented fetal lamb. Am J Obstet Gynecol 1988;159:504-509.

89. Ikeda T, Murata Y, Quuilligan E, et al. Physiologic and histologic changes in near-term fetal lambs exposed to asphyxia by partial umbilical cord occlusion. Am J Obstet Gynecol 1998;178:24-32.

90. Ohyu J, Marumo G, Ozawa H, et al. Early axonal and glial pathology in fetal sheep brains with leukomalacia induced by repeated umbilical cord occlusion. Brain Dev 1999;21:248-252.

91. Matsuda T, Okuyama K, Cho K, et al. Induction of antenatal periventricular leukomalacia by hemorrhagic hypotension in the chronically instrumented fetal sheep. Am J Obstet Gynecol 1999;181:725-730.

92. Duncan J, Cock M, Scheerlinck J, et al. White matter injury after repeated endotoxin exposure in the preterm ovine fetus. Pediatr Res 2002;52:941-949.

93. Dalitz P, Harding R, Rees S, Cock M. Prolonged reductions in placental blood flow and cerebral oxygen delivery in preterm fetal sheep exposed to endotoxin: possible factors in white matter injury after acute infection. J Soc Gynecol Investig 2003;10:283-290.

94. Rees S, Stringer M, Just Y, Hooper S, Harding R. The vulnerability of the fetal sheep brain to hypoxemia at mid-gestation. Devel Brain Res 1997;103:103-118. 
95. Mallard E, Rees S, Stringer M, Cock M, Harding R. Effects of chronic placental insufficiency on brain development in fetal sheep. Pediatr Res 1998;43:262-270.

96. Penning D, Grafe J, Hammond R, Matsuda Y, Patrick J, Richardson B. Neuropathology of the near-term and midgestation ovine fetal brain after sustained in utero hypoxemia. Am J Obstet Gynecol 1994;170:1425-1432.

97. Bagenholm R, Nilsson U, Gotborg C, Kjellmer I. Free radicals are formed in the brain of the fetal sheep during reperfusion after cerebral ischemia. Pediatr Res 1998;43:271-275.

98. Ikeda K, Murata Y, Quilligan EJ, Parer J, Doi S, Park S-D. Brain lipid peroxidation and antioxidant levels in fetal lambs 72 hours after asphyxia from partial umbilical cord occlusion. Am J Obstet Gynecol 1998;178:474-478.

99. Castillo-Melendez M, Chow J, Walker D. Lipid peroxidation, caspase-3 immunoreactivity, and pyknosis in late-gestation fetal sheep brain after umbilical cord occlusion. Pediatr Res 2004;55:864-871.

100. Welin A-K, Sandberg M, Lindblom A, et al. White matter injury following prolonged free radical formation in the 0.65 gestation fetal sheep brain. Pediatr Res 2005;58:100-105.

101. Fraser M, Bennet L, Van Zijl PL, et al. Extracellular amino acids and lipid peroxidation products in periventricular white matter during and after cerebral ischemia in preterm fetal sheep. $\mathrm{J}$ Neurochem 2008;105:2214-2223.

102. Baldwin B, Bell F. The anatomy of the cerebral circulation of the sheep and ox. The dynamic distribution of the blood supplied by the carotid and vertebral arteries to cranial regions. J Anat 1963;97:203-215.

103. Hohimer AR, Chao CR, Bissonnette JM. The effect of combined hypoxemia and cephalic hypotension on fetal cerebral blood flow and metabolism. J Cereb Blood Flow Metab 1991;11:99-105.

104. Iwamoto HS, Kaufman T, Keil LC, Rudolph AM. Responses to acute hypoxemia in fetal sheep at 0.6-0.7 gestation. Am J Physiol 1989;256(3 pt 2):H613-H620.

105. Rurak DW, Richardson BS, Patrick JE, Carmichael L, Homan J. Oxygen consumption in the fetal lamb during sustained hypoxemia with progressive acidemia. 1990;258(5 pt 2):R1108R1115.

106. Richardson BS, Rurak D, Patrick JE, Homan J, Carmichael L. Cerebral oxidative metabolism during sustained hypoxaemia in fetal sheep. 1989;11:37-43.

107. Yan EB, Baburamani AA, Walker AM, Walker DW. Changes in cerebral blood flow, cerebral metabolites, and breathing movements in the sheep fetus following asphyxia produced by occlusion of the umbilical cord. Am J Physiol Regul Integr Comp Physiol 2009;297:R60-R69.

108. Richardson BS. The fetal brain: metabolic and circulatory responses to asphyxia. ClinInvest Med 1993;16:103-114.

109. McClure M, Riddle A, Manese M, et al. Cerebral blood flow heterogeneity in preterm sheep: lack of physiological support for vascular boundary zones in fetal cerebral white matter. J Cereb Blood Flow Metab 2008;28:995-1008.

110. Chao CR, Hohimer AR, Bissonnette JM. Fetal cerebral blood flow and metabolism during oligemia and early postoligemic reperfusion. J Cereb Blood Flow Metab 1991;11:416-423.

111. Nelson MD Jr., Gonzalez-Gomez I, Gilles FH. Dyke Award. The search for human telencephalic ventriculofugal arteries. 1991;12:215222

112. Mayer PL, Kier EL. The controversy of the periventricular white matter circulation: a review of the anatomic literature. 1991;12:223228.

113. Volpe JJ. The structure of blood vessels in the germinal matrix and the autoregulation of cerebral blood flow in premature infants - reply. Pediatrics 2001;108:1050.
114. Buser J, Segovia K, Dean J, et al. Timing of appearance of late oligodendrocyte progenitors coincides with enhanced susceptibility of preterm rabbit cerebral white matter to hypoxia-ischemia. J Cereb Blood Flow Metab 2010;30:1053-1065.

115. Miller S, Ferriero D. From selective vulnerability to connectivity: insights from newborn brain imaging. Trends Neurosci 2009;32:496505.

116. Ment LR, Hirtz D, Huppi PS. Imaging biomarkers of outcome in the developing preterm brain. Lancet Neurol 2009;8:1042-1055.

117. Mathur AM, Neil JJ, Inder TE. Understanding brain injury and neurodevelopmental disabilities in the preterm infant: the evolving role of advanced magnetic resonance imaging. Semin Perinatol 2010;34:57-66.

118. Rutherford MA, Supramaniam V, Ederies A, et al. Magnetic resonance imaging of white matter diseases of prematurity. Neuroradiology 2010;52:505-521.

119. Hope PL, Gould SJ, Howard S, Hamilton PA, Costello AM, Reynolds EO. Precision of ultrasound diagnosis of pathologically verified lesions in the brains of very preterm infants. Dev Med Child Neurol 1988;30:457-471.

120. Schouman-Claeys E, Henry-Feugeas MC, Roset F, et al. Periventricular leukomalacia: correlation between MR imaging and autopsy findings during the first 2 months of life. Radiology 1993;189:59-64.

121. Felderhoff-Mueser U, Rutherford MA, Squier WV, Cox P, Maalouf EF, Counsell SJ, et al. Relationship between MR imaging and histopathologic findings of the brain in extremely sick preterm infants. AJNR Am J Neuroradiol 1999;20:1349-1357.

122. Inder TE, Neil JJ, Kroenke CD, Dieni S, Yoder B, Rees S. Investigation of cerebral development and injury in the prematurely born primate by magnetic resonance imaging and histopathology. Dev Neurosci 2005;27:100-111.

123. Childs AM, Cornette L, Ramenghi LA, et al. Magnetic resonance and cranial ultrasound characteristics of periventricular white matter abnormalities in newborn infants. Clin Radiol 2001;56:647-655.

124. Haynes RL, Billiards SS, Borenstein NS, Volpe JJ, Kinney HC. Diffuse axonal injury in periventricular leukomalacia as determined by apoptotic marker fractin. Pediatr Res 2008;63:656-661.

125. Eklind S, Mallard C, Leverin A, et al. Bacterial endotoxin sensitizes the immature brain to hypoxic-ischaemic injury. Eur $\mathrm{J}$ Neurosci 2001;13:1101-1106.

126. Eklind S, Mallard C, Arvidsson P, Hagberg H. Lipopolysaccharide induces both a primary and a secondary phase of sensitization in the developing rat brain. Pediatr Res 2005;58:112-116.

127. Favrais G, van de Looij Y, Fleiss B, et al. Systemic inflammation disrupts the developmental program of white matter. Ann Neurol 2011;70:550-565.

128. Filan PM, Hunt RW, Anderson PJ, Doyle LW, Inder TE. Neurologic Outcomes in Very Preterm Infants Undergoing Surgery. J Pediatr 2011;160:409-414.

129. Tam EW, Chau V, Ferriero DM, et al. Preterm cerebellar growth impairment after postnatal exposure to glucocorticoids. Sci Transl Med 2011;3:105ra.

130. Brambrink AM, Evers AS, Avidan MS, et al. Isoflurane-induced neuroapoptosis in the neonatal rhesus macaque brain. Anesthesiology 2010;112:834-841.

131. Heine VM, Griveau A, Chapin C, Ballard PL, Chen JK, Rowitch DH. A small-molecule smoothened agonist prevents glucocorticoidinduced neonatal cerebellar injury. Sci Transl Med 2011;3:105ra4.

132. Neubauer AP, Voss W, Kattner E. Outcome of extremely low birth weight survivors at school age: the influence of perinatal parameters on neurodevelopment. Eur J Pediatr 2008;167:87-95.

133. Back SA, Luo NL, Borenstein NS, Levine JM, Volpe JJ, Kinney HC. Late oligodendrocyte progenitors coincide with the developmental window of vulnerability for human perinatal white matter injury. J Neurosci 2001;21:1302-1312. 